\title{
QUANTIDADE E PREÇOS DA BANANA-'PRATA' COMERCIALIZADA NAS CEASAS DO DISTRITO FEDERAL, SÃO PAULO, BELO HORIZONTE E RIO DE JANEIRO, NO PERÍODO DE 1995 A 19991
}

\author{
GENI RODRIGUES FAGUNDES² \& OSVALDO KIYOSHI YAMANISHI ${ }^{3}$
}

\begin{abstract}
RESUMO- A evolução das quantidades e preços médios mensais e anuais da banana-'Prata' comercializada nos entrepostos das CEASAS do Distrito Federal, São Paulo, Belo Horizonte e Rio de Janeiro foi analisada no período de janeiro de 1995 a dezembro de 1999. Os dados foram analisados pelo teste de Tukey, a nível de 5\%. A média da quantidade anual comercializada nas CEASAS do DF, SP, BH e RJ foram, respectivamente, de 831; 1.012; 3.101 e 5.597 t no período analisado. A quantidade média anual comercializada variou significativamente em todas as CEASAS. Os preços médios anuais diferiram significativamente, sendo que em todas as CEASAS estes foram maiores em 1995 e menores em 1997. Não houve diferença significativa na quantidade média mensal comercializada apenas na CEAGESP, já o preço médio mensal diferiu significativamente nas CEASAS do DF, SP e BH. O preço médio anual foi maior na CEASA/ $\mathrm{RJ}(\mathrm{R} \$ 1,01 / \mathrm{Kg})$, que foi $18,8 \% ; 32,9 \%$ e $80,4 \%$ superior quando comparado ao preço praticado na CEASA/ DF, CEAGESP e CEASA/BH, respectivamente.
\end{abstract}

Termos para indexação: banana, CEASAS, quantidade, preços.

\section{QUANTITY AND PRICES OF 'PRATA' BANANA IN THE WHOLESALE MARKET OF FEDERAL DISTRICT, SÃO PAULO, BELO HORIZONTE AND RIO DE JANEIRO IN THE PERIOD OF 1995 TO 1999.}

\begin{abstract}
The variation of the amounts and the annual and monthly average prices of the 'Prata' banana commercialized in the wholesale market (WM) of Federal District (DF), São Paulo (SP), Belo Horizonte (BH) and Rio de Janeiro (RJ) in the period of January of 1995 to December of 1999 were studied. Tukey test at $5 \%$ significance was applied to compare the average amounts and prices. The average of annual amount commercialized in the WM of DF, SP, BH and RJ were, respectively, of $831 ; 1,012 ; 3,101$ and 5,597 tons in the analyzed period. The annual amount commercialized varied significantly in all WM studied. The annual average prices differed significantly, and in all WM these prices were higher in 1995 and lower in 1997. The monthly average amount showed significant difference in the WM of DF, BH and RJ, and the monthly average price not differed in the WM of RJ. The highest annual average price was in the WM of RJ $(\mathrm{R} \$ 1,01 / \mathrm{Kg})$ and it was $18.8 \% ; 32.9 \%$ and $80.4 \%$ higher than the price received in the WM of DF, SP and BH, respectively.
\end{abstract}

Index terms: banana, wholesale market, quantity, prices.

\section{INTRODUÇÃO}

A banana ocupa o segundo lugar dentre as frutíferas cultivadas no Brasil, após os cítricos, apresentando uma produção estimada de 6,0 milhões de toneladas anuais e uma área cultivada próxima de 500.000 hectares (Silva \& Torres Filho,1997).

A bananeira, por ser uma frutífera de clima tropical, apresenta um maior desenvolvimento em condições de temperatura média anual elevada (igual ou superior a $22^{\circ} \mathrm{C}$ ), precipitações pluviométricas anuais acima de $1.200 \mathrm{~mm}$ e bem distribuídas. Portanto, a época de colheita da banana está relacionada com as condições climáticas e com os tratos culturais (Manica, 1997). Segundo este autor, a bananeira apresenta um ciclo mais curto durante o período quente e úmido e mais longo no período frio e seco. Sendo assim, os cachos desenvolvem-se mais rapidamente nos meses quentes, causando maior oferta do produto e, conseqüentemente, menores preços. Já nos meses frios, ocorre o contrário, pois o desenvolvimento dos cachos é retardado, reduzindo a oferta e provocando maiores preços.

A banana é caracterizada pelo comércio de vizinhança e, por ser um produto muito perecível, é importante que sua comercialização seja rápida, racional e com cuidados para reduzir as perdas e, ainda, para que o produto chegue ao seu destino em boas condições. A qualidade da fruta é essencial não apenas para a exportação, mas também para o mercado interno, pois muitos supermercados já diferenciam os preços de produtos perecíveis, caso das frutas, através da marca e, obviamente, da

1 (Trabalho 238/2000). Recebido: 27/10/2000. Aceito para publicação: 31/07/2001.

2 Eng. Agr. MSc., Faculdade de Agronomia e Medicina Veterinária da Universidade de Brasília (UnB). Bolsista DTI do CNPq. E-mail: genifagundes@bol.com.br

3 Eng. Agr. PhD, Professor Adjunto II da Faculdade de Agronomia e Medicina Veterinária da UnB. Campus Universitário Darcy Ribeiro, Cx. Postal 04508, CEP 70.910-970, Brasília-DF. E-mail: kiyoshi@unb.br 
qualidade (Anuário Estatístico, 1998).

A estrutura de comercialização da banana no Brasil consiste, quase sempre, na participação direta do produtor e do comprador, sendo que as CEASAS apenas centralizam a distribuição dos produtos nas capitais dos estados.

Com relação à formação do preço, a mesma depende dos seguintes fatores: procura, qualidade e quantidade ofertada. No Brasil, a quantidade de banana ofertada é grande; no entanto, a qualidade do produto, em determinados locais, ainda é baixa, contribuindo para que o preço, principalmente ao nível de produtor, seja baixo. A baixa qualidade da banana e a adoção de estruturas precárias de produção e comercialização são consideradas entraves à exportação da fruta pelo País (Pizzol e Eleutério, 2000).

Alguns produtores de bananas já estão se conscientizando de que a qualidade é um dos principais fatores na formação do preço, e por isso estão investindo em novas tecnologias para o manejo pós-colheita desses frutos. No entanto, ainda são poucos aqueles que utilizam tecnologias adequadas aos padrões internacionais.

Com o advento do Plano Real (estabilização da moeda brasileira) e também com a tendência moderna de uma dieta mais saudável, houve um aumento do consumo per capita de frutas. No Brasil, o consumo per capita de banana evoluiu de $21,5 \mathrm{~kg} /$ hab/ano no triênio 73/75 para 24,4 kg/hab/ano no triênio 95/96 (Mascarenhas, 1999). Este incremento no consumo da banana contribuiu para um aumento dos preços, seguindo a lei da oferta e procura.

Portanto, é muito importante o conhecimento da variação estacional de preço e quantidade, para a orientação dos bananicultores, dos comerciantes e até dos consumidores, pois as oscilações de preços têm efeito na elaboração do orçamento familiar e também no fluxo de caixa dos produtores (Perez et al., 1995).

O presente trabalho teve como objetivo verificar a variação das quantidades e preços da banana- 'Prata' comercializada nas CEASAS do Distrito Federal (DF), São Paulo (SP), Belo Horizonte (BH) e Rio de Janeiro (RJ).

\section{MATERIAL E MÉTODOS}

Neste trabalho, foram utilizados dados dos levantamentos mensais da quantidade de banana- 'Prata' comercializada nas CEASAS do DF, SP, BH e RJ, no período de julho de 1994 a dezembro de 1999, registrados pelas respectivas CEASAS.

As quantidades de banana-'Prata' mensalmente comercializadas são o somatório das quantidades diárias, enquanto as quantidades anuais são a média dos meses no referido ano. Estas quantidades, anteriormente em quilograma, foram convertidas para tonelada.

Os preços médios mensais, corrigidos pelo índice de inflação mensal, foram obtidos também através de levantamentos feitos pelas respectivas CEASAS, sendo apresentados em R $\$ /$ $\mathrm{kg}$. Os preços médios anuais representam a média dos preços médios mensais no referido ano, em valores nominais.

Para comparação das quantidades e preços médios anuais e mensais, foi aplicado o teste de Tukey, ao nível de 5\% probabilidade.

\section{RESULTADOS E DISCUSSÃO}

$\mathrm{Na}$ Tabela 1, encontram-se os dados referentes às quantidades e preços médios anuais da banana- 'Prata' comercializada nas CEASAS do DF, SP, BH e RJ, no período de janeiro de 1995 a dezembro de 1999. Verificou-se que, até 1997, houve um aumento significativo na quantidade média de banana comercializada nas 4 CEASAS analisadas, decrescendo em 1998 e voltando a crescer em 1999. A quantidade média de banana comercializada foi maior no ano de 1999 nas CEASAS do DF, BH e RJ, enquanto, na CEAGESP, esta foi maior em 1997. O ano de 1995 apresentou menor volume comercializado.

Os preços médios anuais diferiram estatisticamente, sendo que, em todas as CEASAS, estes foram maiores em $1995 \mathrm{e}$ menores em 1997 (exceto na CEASA/RJ). Isso mostra que, em 1995, o preço seguiu a lei da oferta e procura; já em 1997, o comportamento foi atípico. Esta variação pode também ser devida às adversidades climáticas nas zonas produtoras.

Na CEAGESP, no período analisado, a média de comercialização da banana-'Prata' foi de 1.012 toneladas anuais, bastante inferior quando comparada com o volume comercializado nas CEASAS de BH e RJ. Isto se deve ao fato de a banana'Prata' representar apenas $17,4 \%$ da banana comercializada no Estado de São Paulo, sendo a banana-'Nanica' responsável por $72 \%$, em contraste com as CEASAS do RJ, BH e DF, onde a banana-'Prata' responde por $77,8 \% ; 58,7 \%$ e $40,9 \%$, respectivamente, do volume total comercializado de banana (Mascarenhas, 1999). O preço médio anual foi maior na CEASA/ $\mathrm{RJ}(\mathrm{R} \$ 1,01 / \mathrm{kg})$, que foi $18,8 \% ; 32,9 \%$ e $80,4 \%$ superior quando comparado ao preço praticado nas CEASAS do DF, SP e BH, respectivamente. $\mathrm{O}$ fato de a média dos preços anuais praticados nas CEASAS de $\mathrm{SP}$ e $\mathrm{BH}$ terem sido inferiores à média dos preços das CEASAS do DF e RJ, pode ser atribuído ao fato de estes entrepostos estarem situados próximos das principais zonas produtoras de banana do Brasil. No caso de São Paulo, dados mostram que cerca de $70 \%$ da banana-'Prata' é oriunda de municípios do próprio Estado (FrutiSéries 6, 1998).

As quantidades e os preços médios mensais da banana'Prata' comercializada nas CEASAS do DF, SP, BH e RJ estão na Tabela 2. Houve diferença significativa na quantidade média mensal comercializada em todas as CEASAS. A quantidade de banana-'Prata' comercializada nas quatro CEASAS tendeu a ser maior no período de setembro a janeiro, concordando com Perez et al. (1995) que concluíram que as condições climáticas (chuva e temperatura) afetam tanto a oferta quanto a demanda de banana. Segundo estes autores, os maiores índices de precipitação pluviométrica e temperatura elevada aceleram a formação dos cachos, aumentando a quantidade de banana enviada ao mercado e provocando a redução dos preços e vice-versa. Conte et al. (1994) observaram que a quantidade de banana comercializada na CEASA/RS, no período de 1985 a 1995, foi maior no mês de março, a qual foi estatisticamente superior ao volume comercializado no período de junho a outubro e semelhante à quantidade comercializada nos demais meses.

A quantidade média mensal comercializada teve relação inversa e direta com o preço médio mensal para as CEASAS do DF e BH, já as demais apresentaram, em alguns meses, um comportamento atípico, ou seja, alta oferta e altos preços.

Observou-se que houve variação estatística do preço 
TABELA 1 - Quantidade (t) e preço médio anual (R\$) do kg da banana-'Prata' comercializada nas CEASAS do Distrito Federal, São Paulo, Belo Horizonte e Rio de Janeiro, no período de janeiro de 1995 a dezembro de 1999.

\begin{tabular}{|c|c|c|c|c|c|c|c|c|}
\hline \multirow[b]{2}{*}{ A no } & \multicolumn{2}{|c|}{ CEASA /D F } & \multicolumn{2}{|c|}{ C E A G E S P } & \multicolumn{2}{|c|}{ C E A S A / B H } & \multicolumn{2}{|c|}{ C E A S A / R J } \\
\hline & $Q$ u ant. & Preço & $Q$ u ant. & P reço & Quant. & Preço & Quant. & Preço \\
\hline 1995 & $747 \mathrm{c}$ & $1,10 \mathrm{a}$ & $458 \mathrm{~b}$ & $1,01 \mathrm{a}$ & $2.255 \mathrm{e}$ & $0,80 \mathrm{a}$ & $4.811 \mathrm{~d}$ & $1,46 \mathrm{a}$ \\
\hline 1996 & $745 \mathrm{c}$ & $0,92 \mathrm{~b}$ & $1.231 \mathrm{a}$ & $0,73 \mathrm{~b}$ & $2.685 \mathrm{~d}$ & $0,55 \mathrm{~b}$ & $5.545 \mathrm{bc}$ & $1,11 \mathrm{~b}$ \\
\hline 1997 & $892 \mathrm{ab}$ & $0,68 \mathrm{~d}$ & $1.399 \mathrm{a}$ & $0,65 \mathrm{~b}$ & $3.581 \mathrm{~b}$ & $0,36 \mathrm{c}$ & $6.082 \mathrm{ab}$ & $0,83 \mathrm{~cd}$ \\
\hline 1998 & $822 \mathrm{bc}$ & $0,82 \mathrm{c}$ & $894 \mathrm{ab}$ & $0,72 \mathrm{~b}$ & $3.142 \mathrm{c}$ & $0,56 \mathrm{~b}$ & $5.261 \mathrm{~cd}$ & $0,95 \mathrm{bc}$ \\
\hline 1999 & 949 a & $0,74 \mathrm{~cd}$ & $1.076 \mathrm{a}$ & $0,71 \mathrm{~b}$ & $3.843 \mathrm{a}$ & $0,51 \mathrm{~b}$ & $6.284 \mathrm{a}$ & $0,68 \mathrm{~d}$ \\
\hline Média & 831 & 0,85 & 1012 & 0,76 & 3101 & 0,56 & 5597 & 1,01 \\
\hline
\end{tabular}

* Médias seguidas de mesma letra na coluna não diferem significativamente entre si, pelo teste de Tukey, ao nível de $5 \%$ de probabilidade.

TABELA 2 - Quantidade (t) e preço médio mensal (R\$) do kg da banana-'Prata' comercializada nas CEASAS do Distrito Federal, São Paulo, Belo Horizonte e Rio de Janeiro, no período de janeiro de 1995 a dezembro de 1999.

\begin{tabular}{|c|c|c|c|c|c|c|c|c|}
\hline \multirow[b]{2}{*}{ M ês } & \multicolumn{2}{|c|}{ CEASA / D F } & \multicolumn{2}{|c|}{ CEAGESP } & \multicolumn{2}{|c|}{ CEASA/BH } & \multicolumn{2}{|c|}{ CEASA $\mathrm{A}$ / $\mathrm{J}$} \\
\hline & $Q$ uant. & Preço & $Q$ u ant. & Preço & Quant. & Preço & Quant. & Preço \\
\hline Jan. & $821 \mathrm{abcd}$ & $0,87 \mathrm{abc}$ & $1021 \mathrm{a}$ & $0,75 \mathrm{ab}$ & $2649 \mathrm{ef}$ & $0,59 \mathrm{abcd}$ & $5557 \mathrm{abc}$ & $1,01 \mathrm{a}$ \\
\hline Fev. & $779 \mathrm{bcd}$ & $0,88 \mathrm{abc}$ & $853 \mathrm{a}$ & $0,79 a b$ & $2742 \mathrm{def}$ & $0,61 \mathrm{abc}$ & $4684 \mathrm{c}$ & $1,06 \mathrm{a}$ \\
\hline M ar & $909 a b c$ & $0,86 \mathrm{abc}$ & $958 \mathrm{a}$ & $0,78 \mathrm{ab}$ & $3205 \mathrm{bcd}$ & $0,59 \mathrm{abcd}$ & $6063 \mathrm{ab}$ & $0,95 \mathrm{a}$ \\
\hline Abr. & $836 a b c d$ & $0,88 \mathrm{abc}$ & $1151 \mathrm{a}$ & $0,78 \mathrm{ab}$ & $2825 \mathrm{def}$ & $0,63 \mathrm{abc}$ & $5548 \mathrm{abc}$ & $1,02 \mathrm{a}$ \\
\hline $\mathrm{M}$ a io & $794 \mathrm{abcd}$ & $0,90 \mathrm{abc}$ & $875 \mathrm{a}$ & $0,81 \mathrm{ab}$ & $2818 \mathrm{def}$ & $0,64 \mathrm{ab}$ & $5427 \mathrm{abc}$ & $1,07 \mathrm{a}$ \\
\hline Jun. & $699 \mathrm{~d}$ & $0,92 \mathrm{a}$ & $1146 \mathrm{a}$ & $0,83 \mathrm{ab}$ & $2514 \mathrm{f}$ & $0,68 \mathrm{a}$ & $4922 \mathrm{bc}$ & $1,14 \mathrm{a}$ \\
\hline Ju 1 . & $709 \mathrm{~d}$ & $0,93 \mathrm{a}$ & $847 \mathrm{a}$ & $0,84 \mathrm{a}$ & $2665 \mathrm{ef}$ & $0,66 \mathrm{ab}$ & $5329 \mathrm{abc}$ & $1,15 \mathrm{a}$ \\
\hline Ago. & $757 \mathrm{~cd}$ & $0,91 \mathrm{ab}$ & $943 \mathrm{a}$ & $0,81 \mathrm{ab}$ & $3038 \mathrm{cde}$ & $0,60 \mathrm{abcd}$ & $5730 \mathrm{abc}$ & $1,09 \mathrm{a}$ \\
\hline Set. & $858 \mathrm{abcd}$ & $0,89 \mathrm{abc}$ & $1122 \mathrm{a}$ & $0,76 a b$ & $3537 \mathrm{ab}$ & $0,49 \mathrm{abcd}$ & $5566 \mathrm{abc}$ & $0,98 \mathrm{a}$ \\
\hline Out. & $941 \mathrm{ab}$ & $0,75 \mathrm{abc}$ & $1129 \mathrm{a}$ & $0,72 \mathrm{ab}$ & $3862 \mathrm{a}$ & $0,39 \mathrm{~cd}$ & $6224 \mathrm{a}$ & $0,92 \mathrm{a}$ \\
\hline Nov. & $958 \mathrm{a}$ & $0,70 \mathrm{c}$ & $1059 \mathrm{a}$ & $0,68 \mathrm{ab}$ & $3836 \mathrm{a}$ & $0,36 \mathrm{~d}$ & $6011 \mathrm{ab}$ & $0,86 \mathrm{a}$ \\
\hline $\mathrm{Dez}$ & $911 \mathrm{abc}$ & $0,72 \mathrm{bc}$ & $1032 \mathrm{a}$ & $0,61 \mathrm{~b}$ & $3522 \mathrm{abc}$ & $0,42 \mathrm{bcd}$ & $6098 \mathrm{ab}$ & $0,80 \mathrm{a}$ \\
\hline
\end{tabular}

* Médias seguidas de mesma a letra na coluna não diferem significativamente entre si, pelo teste de Tukey, ao nível de $5 \%$ de probabilidade.

médio mensal da banana-'Prata' comercializada nas CEASAS do DF, SP e BH no período analisado. Os meses de junho e julho apresentaram maior preço médio mensal em todas as CEASAS, mostrando que, no período onde geralmente as temperaturas são mais baixas, a oferta da banana é menor, provocando assim aumento dos preços. O preço médio, em todas as CEASAS, foi menor nos meses de outubro, novembro e dezembro, que normalmente coincide com altas temperaturas e pluviosidade, favorecendo o desenvolvimento da cultura da banana e, conseqüentemente, aumentando a oferta do produto.

É importante, portanto, que os produtores de banana estejam preparados para colocar seu produto no mercado e fazer a negociação de sua produção, buscando deslocá-la para épocas de melhores preços. Uma das formas de se deslocar a produção do bananal seria através do manejo das touceiras pela eliminação programada das brotações e com um adequado manejo nutricional e fitossanitário da cultura.

Sugere-se que os produtores adotem novas tecnologias de cultivo para melhorar a qualidade do fruto e aumentar a eficiência na produção e na comercialização, reduzindo perdas e custos, e aumentando receitas.

\section{CONCLUSÕES}

A análise dos dados permite concluir que:
1. As quantidades médias de banana-'Prata' comercializadas nas CEASAS estudadas apresentaram variações significativas. Já a quantidade média mensal foi estável apenas na CEAGESP.

2. O preço médio anual da banana-'Prata' praticado nas CEASAS do DF, SP, BH e RJ foi maior em 1995 e menor em 1997 (exceto na CEASA/RJ).

3. O preço médio mensal da banana-'Prata' não diferiu significativamente apenas na CEASA/RJ, sendo maior nos meses de junho e julho.

\section{REFERÊNCIAS BIBLIOGRÁFICAS}

ANUÁRIO ESTATÍSTICO DA AGRICULTURA BRASILEIRA AGRIANUAL 98. São Paulo: FNP, Consultoria \& Comércio. 1998. p.132.

BRASIL. Ministério do Meio Ambiente, dos Recursos Hídricos e da Amazônia Legal. Banana. Brasília. 1998. v.6, 4p. (Frutiséries)

CEASA. Quantidades e preços médios mensais da banana-Prata comercializada na CEASA/DF. Brasília, 1994/1998.

CEASA. Quantidades e preços médios mensais da banana-Prata comercializada na CEAGESP. São Paulo, 1994/1998.

CEASA. Quantidades e preços médios mensais da banana-Prata 
comercializada na CEASA/BH. Belo Horizonte, 1994/1998.

CEASA. Quantidades e preços médios mensais da banana-Prata comercializada na CEASA/RJ. Rio de Janeiro, 1994/1998.

CONTE, A.; KRAUSE, C.A.; RECH, N.L.; RODEL, M.F.; RODRIGUES, V.C.; MANICA, I. Origem, quantidade e preço da banana comercializada na CEASA/RS de janeiro de 1985 a dezembro de 1994.

INSTITUTO DE PLANEJAMENTO E ECONOMIA AGRÍCOLA DE SANTA CATARINA. Banana. Florianópolis, 1995. 103p. (Estudo de economia e mercado de produtos agrícolas, 2).

MANICA, I. Fruticultura tropical 4, Banana. Porto Alegre: Cinco Continentes, 1997. 485p.

MASCARENHAS, G.C.C. Viabilidade mercadológica e financeira da atividade produtora de banana na Região de Ilhéus-
Itabuna. 1997. 171f. Tese (mestrado) - Universidade Federal de Viçosa,

MASCARENHAS, G.C.C. Banana: Comercialização e Mercados. Informe Agropecuário, Belo Horizonte, v.20, n.196, p.97-108, 1999.

PEREZ, L. H.; PINO, F. A.; FRANCISCO, V. L. F. dos S. Preço recebido pelo produtor de banana no Estado de São Paulo: uma análise de séries temporais. Agricultura em São Paulo, São Paulo, v.42, n.1, p.133-141, 1995.

PIZZOL, J.S.S.de; ELEUTÉRIO, R.C. Fruticultura: participação do Brasil no mercado externo de bananas. Preços Agrícolas, São Paulo, v. 14, n.162, p.40-41, 2000.

SILVA, J. da; TORRES FILHO, P. Aspectos socioeconômicos. In: A CULTURA DA BANANA: ASPECTOS TÉCNICOS, SOCIOECONÔMICOS E AGROINDUSTRIAIS. Brasília: EMBRAPA-CNPMF, 1997. 585p. 